\title{
Gibt es einen wissenschaftlichen Konsens zur Wirtschaftlichkeit nachhaltiger Immobilien?
}

\section{ZIC̈ gif}

\author{
Franz Fuerst • Ben Dalton
}

Online publiziert: 25. Oktober 2019

(C) Der/die Autor(en) 2019

Zusammenfassung Es besteht ein klarer wissenschaftlicher und gesellschaftlicher Konsens über die Schlüsselrolle, die Gebäuden und der gebauten Umwelt bei der Reduzierung von Treibhausgasemissionen und Ressourcenverbrauch zukommt. Unklar ist jedoch, inwieweit Umweltziele und ökonomische Rentabilität im Immobilienbereich miteinander einhergehen. Dieser Artikel analysiert 42 internationale Studien zur Rentabilitaet von Immobilien mit Nachhaltigkeitszertifikaten anhand einer Metaanalyse und kommt zu dem Schluss, dass diese Gebäude zumindest ein moderater Preis- und Mietpraemium erzielen. Obwohl es Hinweise darauf gibt, dass Studien, die positive Ergebnisse berichten, mit höherer Wahrscheinlichkeit veröffentlicht wurden, liefern diese Studien dennoch einen Anhhaltspunkt dafür, dass nachhaltiges Bauen und Sanieren vom Markt als wirtschaftlich sinnvoll erkannt und entsprechend bepreist wird. Dabei ist allerdings zu bedenken, dass der wirtschaftliche Erfolg umweltgerechter Immobilieninvestments unter anderem auch davon abhängt, inwieweit Marktmechanismen etabliert werden können, die ein effiziente Verteilung von Risiko und Rendite ermöglichen, wie zum Beispiel grüne Mietverträge oder EnergiesparContracting.

Schlüsselwörter Energieeffizienz · Gebäudezertifikate $\cdot$ Metaanalyse $\cdot$ Green Premium · Immobilienmärkte

F. Fuerst $(\bowtie) \cdot$ B. Dalton University of Cambridge, Cambridge, Großbritannien E-Mail: ff274@cam.ac.uk 


\title{
Is there a scientific consensus on the economic viability of sustainable buildings?
}

\begin{abstract}
There is a clear consensus among scientists and the larger public on the importance of buildings and the built environment in reducing carbon emissions and resource consumption. It is not clear, however, to what extent environmental and economic objectives are aligned, i.e. if sustainable and energy-efficient buildings generally also make for profitable investments. The present paper analyses 42 international studies on the profiftability of sustainable buildings using a metaanalysis framework and concludes that at least a moderate price and rental premium exsists. Despite signs of publication bias in the existing studies, i.e. positive results are more likely to be published than negative or inconclusive findings, commercial and residential real estate markets appear to consider sustainable construction and refurbishment an economically sound endeavour. It is worth noting that the feasibility of individual green investments depends, among others, on market mechanisms for sharing risks and rewards efficiently, for example through green leases or energy performance contracting.
\end{abstract}

Keywords Energy efficiency · Building certificates · Metaanalysis · Green premium $\cdot$ Real estate markets

Nachhaltige Immobilieninvestments erfreuen sich großer Beliebtheit. Weltweit sind bereits $27 \%$ aller Bau- und Immobilienfachkräfte mehrheitlich in Projekten engagiert, bei denen Nachhaltigkeit eine Rolle spielt und gehen, davon aus, dass dieser Anteil bis 2021 auf $47 \%$ ansteigen wird (Dodge Data und Analytics 2018). Interessanterweise liegt dieser Anteil in Deutschland mit $13 \%$ bzw. 35\% allerding deutlich niedriger als in anderen Ländern mit vergleichbarem Entwicklungsstand, wie etwa Australien, Großbritannien oder selbst den USA, die global zu den Staaten mit dem höchsten Pro-Kopf-Ausstoss an Treibhausgasen zählen. Ob dies lediglich einen Nachholbedarf dieser Länder widerspiegelt oder eher auf eine mangelnde Zukunftsorientierung der deutschen Bau- und Immobilienbranche hinweist, ist empirisch nur schwer zu erforschen. Im globalen Rahmen wird jedoch zunehmend deutlich, dass die derzeitigen Anstrengungen im Bereich der gebauten Umwelt nicht ausreichen, um das 2 Grad-Ziel der Erderwärmung zu erreichen. Als eines der grössten Hindernisse werden die Kapitalintensität der erforderlichen Massnahmen sowie die angebliche mangelnde Zahlungsbereitschaft von Gewerbe- und Wohnungsmietern genannt. Die vorliegende Studie geht daher der Frage nach, inwieweit sich aus der wissenschaftlichen Literatur Hinweise auf die Wirtschaftlichkeit nachhaltiger Gebäude über eine hedonische Analyse von Kaufpreisen und Mieten ableiten lassen. ${ }^{1}$

\footnotetext{
1 Die Ergebnisse der in diesem Artikel dargestellten Metaanalyse wurden in englischer Sprache im Routledge Handbook of Sustainable Real Estate veroeffentlicht. Der vorliegende Artikel greift diese Ergebnisse auf und geht zudem auf die Situation in Deutschland ein.
} 


\section{Grüne Gebäudezertifikate in der Nachhaltigkeitsdebatte}

Bevor die Frage der Wirtschaftlichkeit untersucht werden kann, ist jedoch zunächst zu klären, welche Gebäude als nachhaltig bezeichnet werden können. Der Begriff Nachhaltigkeit umfasst zahlreiche Aspekte und kann, je nach Definition und Zweck einer Bewertung, breiter oder enger gefasst werden. Im Kontext des Wohnungmarktes bedeutet dies beispielsweise, dass einige Definitionen die Qualität des Gebäudes hinsichtlich Energie- und Wasserverbrauch berücksichtigen, während andere Bewertungsraster auch Mobilität und Standort oder etwa die Emissionen der Baumaterialien über den gesamten Lebenszyklus einbeziehen. Wohl nicht zuletzt aufgrund dieser Komplexität der zu berücksichtigenden Dimensionen sowie der Tatsache, dass Nachhaltigkeit bei Gebäuden dem nicht fachkundigen Betrachter weitgehend verborgen bleibt, haben verbindliche private und staatliche Umweltbewertungssysteme in mehreren Ländern Anklang gefunden. Öko-Labels wie DGNB in Deutschland, BREEAM in Großbritannien, Green Star in Australien und LEED in den USA sind einige bekannte Beispiele für Zertifizierungssystem, die parallel zu gesetzlichen Bauvorschriften entwickelt wurden. Obwohl diese Label-Systeme unterschiedliche Schwerpunkte in den verschiedenen Nachhaltigkeitskategorien setzen, handelt es sich im Kern um marktbasierte Initiativen, die über die gesetzlichen Anforderungen und nationalen Bauvorschriften hinausgehen (Hamedani and Huber 2012). Es liegt also die Frage nahe, ob die Auszeichung eines Gebäudes mit einem Label Grundlage für eine ,grüne Prämie“ bei Verkauf oder Vermietung schaffen kann. Neben der Frage nach der finanziellen Outperformance zertifizierter Gebäude ist von wissenschaftlichen Studien auch zu klären, inwieweit die Zertifizierung an sich oder die darin zum Ausdruck gebrachte Energiesparsamkeit und weitere messbare Nachhaltigkeitseigenschaften eventuelle Markteffekte erzeugen.

Die internationale Literatur zur Umwelt- und Finanzperformance nachhaltiger Gebäude ist in den letzten zehn Jahren stark angewachsen. Zwei der meistzitierten Studien in diesem Feld wurden von Eichholtz et al. (2010) und Fuerst und McAllister (2011c) vorgelegt und liefern empirische Belege aus dem US-amerikanischen Büromarkt, dass eine grüne Prämie tatsächlich nachweisen lässt. Auch im Bereich der Wohnimmobilien wurden zahlreiche Studien veröffentlicht mit empirischen Untersuchungen aus Spanien (Taltavull de La Paz et al. 2019), den Niederlanden (Brounen und Kok 2011), Finnland (Fuerst et al. 2016), Japan (Fuerst und Shimizu 2016) und Singapur (Deng et al. 2012).

Für den deutschen Wohnimmobilienmarkt sind zumindest zwei jüngere Studien vorhanden, die sich mit den Preiseffekten des Energieausweises beschäftigen. Kholodilin et al. (2017) untersuchen Transaktionen auf dem Berliner Wohnungsmarkt und finden empirische Belege, dass energieeffizientere Wohnungen höhere Verkaufspreise erzielen, selbst wenn man andere Faktoren wie Lage, Alter und Ausstattung konstant hält. Allerdings stellen die Autoren auch fest, dass die implizite Zahlungsbereitschaft von Mietern unter dem Niveau der potentiellen einsparbaren Energiekosten liegt. Cajias et al. (2019) kommen zu ähnlichen Ergebnissen in ihrer Untersuchung von über 400 lokalen Wohnungsmärkten in Deutschland und stellen ausserdem fest, dass Wohnungen, die Defizite bei der Energieeffizienz aufweisen, längere Zeit auf dem Markt verbringen und somit illiquider sind. 


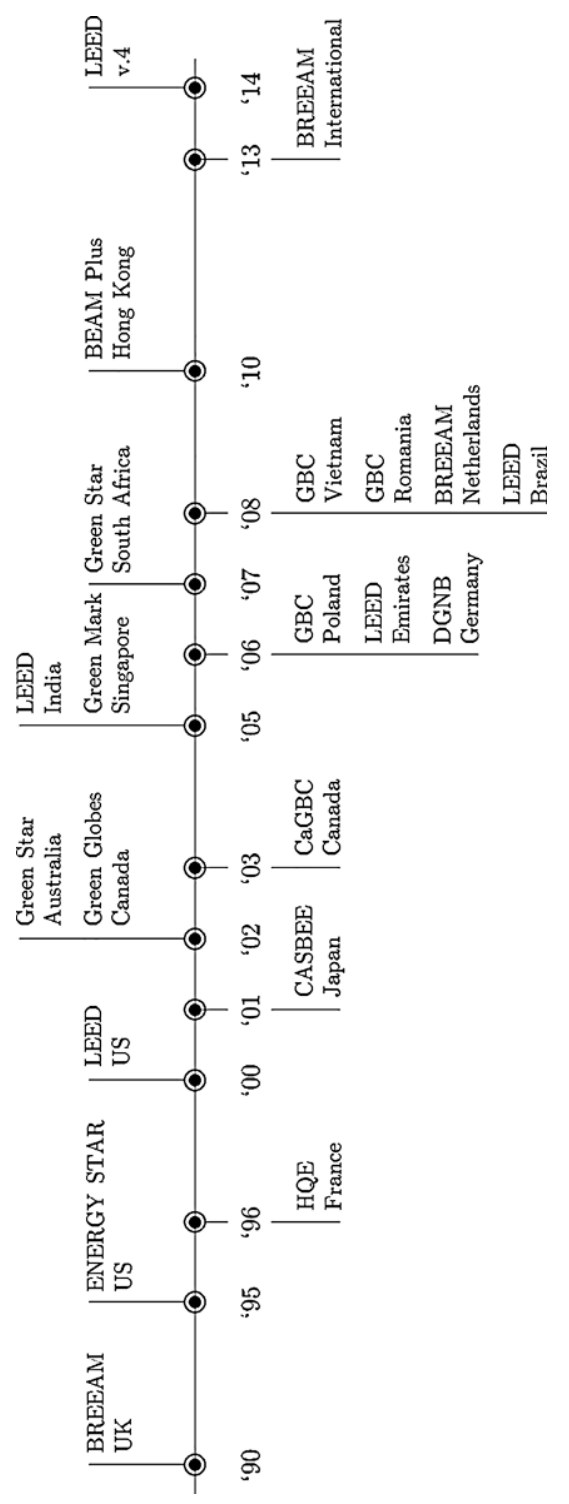

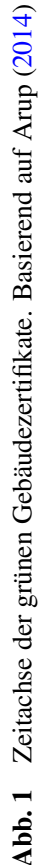


Tab. 1 Suchbegriffe der Metaanalyse

\begin{tabular}{|c|c|c|c|c|}
\hline \multicolumn{3}{|l|}{ Begriffsgruppen } & & \multirow[t]{2}{*}{ Suchsyntax } \\
\hline Energy-efficient & Real estate & Price & Premium & \\
\hline $\begin{array}{l}\text { Green, environ*, eco, } \\
\text { responsible, RPI, } \\
\text { sustainab*, energy- } \\
\text { efficient, energy effi- } \\
\text { cient, energy rating, } \\
\text { energy performance, } \\
\text { energy certificat*, } \\
\text { environ* rating, en- } \\
\text { viron*, performance, } \\
\text { environ* certificate, } \\
\text { EPC, performance } \\
\text { certificat*, eco-label, } \\
\text { BREEAM, LEED, } \\
\text { Energy Star }\end{array}$ & $\begin{array}{l}\text { Real } \\
\text { estate, } \\
\text { property, } \\
\text { properties, } \\
\text { building\$ }\end{array}$ & $\begin{array}{l}\text { Price\$, va- } \\
\text { lue\$, rent\$, } \\
\text { transacti- } \\
\text { on\$, sale\$ }\end{array}$ & $\begin{array}{l}\text { Premium\$, } \\
\text { capitali?ed, } \\
\text { capitali?e }\end{array}$ & $\begin{array}{l}\text { ("green" OR environ* OR } \\
\text { "eco" OR "responsible" } \\
\text { OR "RPI" OR sustainab* } \\
\text { OR "'energy-efficient" OR } \\
\text { (("energy" OR environ*) } \\
\text { AND (efficien* OR "rating" } \\
\text { OR "performance" OR } \\
\text { certificat*)) OR "EPC" } \\
\text { OR performance certi- } \\
\text { ficat* OR "eco-label" } \\
\text { OR "BREEAM" OR } \\
\text { "LEED" OR "Energy Star") } \\
\text { AND ("real estate" OR } \\
\text { "property" OR "properties" } \\
\text { OR building\$) AND } \\
\text { ("price" OR "value" AND } \\
\text { "premium" OR capitali?ed } \\
\text { OR capitali?e) }\end{array}$ \\
\hline
\end{tabular}

Wie sich Energieeffizienz und Nachhaltigkeit bei Gebäuden auf die Rentabilität einer Immobilieninvestition auswirkt, könnte somit mittlerweile als weitgehend erforscht gelten. Die empirischen Studien zu diesem Thema sind jedoch häufig geographisch und zeitlich begrenzt. Von wenigen Ausnahmen abgesehen (Dalton und Fuerst 2018; Fizaine et al. 2018) gibt es kaum Versuche, die Studien zur Wirtschaftlichkeit nachhaltiger Gebäude zu konsolidieren und die einzelnen Studien in einen größeren Zusammenhang zu stellen.

Nach unserem Kenntnisstand wurde nur eine Metaanalyse der finanziellen Performance nachhaltiger Gebäude im Immobilienbereich vorgenommen. Die Studie von Brown und Watkins (2015) analysiert die grünen Prämien für umweltzertifizierte Gebäude anhand einer Auswahl von 20 Studien, hauptsächlich des US-amerikanischen Wohnimmobilienmarktes.. Die anderen, von Experten begutachteten Papiere, die eine systematische Überprüfung nachhaltiger Immobilien vornehmen, tun dies häufig in einem viel breiteren Kontext. Ein Artikel von Zhang (2015) verwendet relativ weitgefasste Suchbegriffe, um allgemeine Themen in der Literatur zu bestimmen, anstatt auf enge Suchbegriffe zu fokussieren, die genaueren Aufschluss zum Thema Rentabilität nachhaltiger Immobilien geben könnten. Darüber hinaus konzentriert sich die Überprüfung auf den chinesischen Markt, der aufgrund seiner speziellen Merkmale nur sehr bedingt mit anderen Märkten zu vergleichbar ist. Die vorliegende Studie liefert hingegen einen breiten Überblick zu den wichtigsten internationalen Märkten und geht dann auf die Situation in Deutschland ein. Wie aus Abb. 1 hervorgeht, wurden die ersten grünen Gebäudezertifikate bereits in den 1990er Jahren in Großbritannien, den USA und Frankreich etabliert. Einige dieser Zertifizierungen, beispielsweise BREEAM und LEED, expandierten in den folgenden Jahren auch in internationale Märkte beziehungsweise wurden an die Gegebenheiten anderer Märkte angepasst (z. B. BREEAM Niederlande). Dadurch ergibt sich in diesen Ländern zuweilen eine Konkurrenzsituation zwischen den einheimischen 


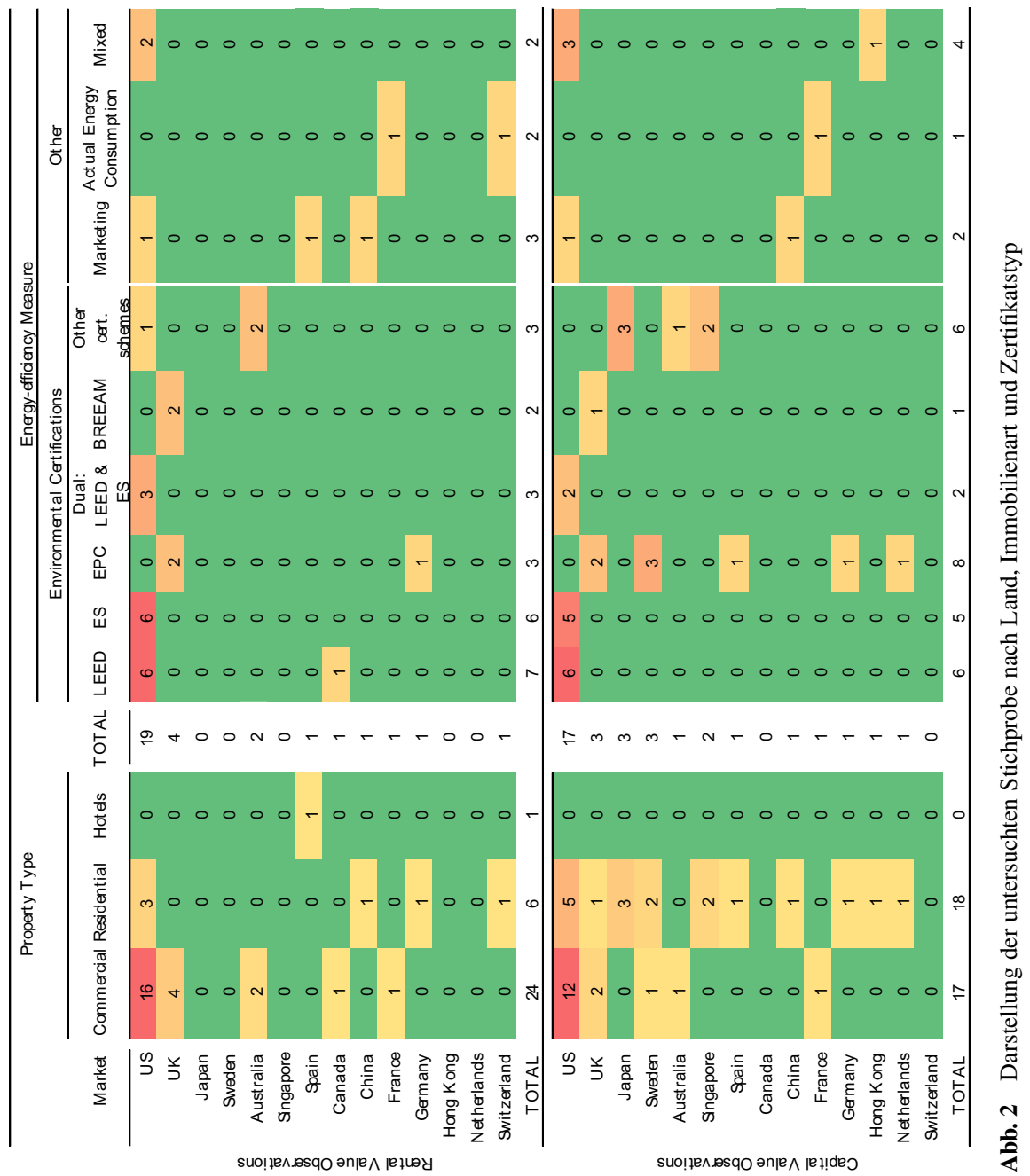


Zertifizierungen und den internationalen Labels, die allerdings in einigen Fällen bei größeren Objekten durch eine Doppelzertifizierung gemindert wird.

\section{Methodik}

Die Methodik des vorliegenden Studie folgt dem von Klewitz und Hansen (2014) vorgeschlagenen Verfahren, wie in Tab. 1 dargestellt. Im folgenden werden die einzelnen Schritte beschrieben:

Schritt 1 Zunächst erfolgt eine Suche in der englischsprachigen Literatur auf Basis von Schlüsselwörtern, die sich auf vier Komponenten (Suchgruppen) beziehen: Energieeffizienz, Immobilien, Preis, Prämie. Insgesamt wurden 32 Schlüsselwörter zur Beschreibung der vier Suchgruppen berücksichtigt. Die beispielhafte Suchsyntax in Tab. 1 ergibt nur Studien, die mindestens ein Wort aus jeder Suchgruppe enthalten.

Schritt 2 Eine systematische Überprüfung der Literatur in einer Metaanalyse ist jedoch nicht automatisch methodisch valide. Es gibt vielmehr eine Reihe von Validitätsfallen, die die Gültigkeit der Analyse gefährden (Borenstein et al. 2011; Hopewell et al. 2009). Zum Beispiel können die tatsächlichen Effekte durch den sogenannten Publikationsbias verzerrt werden, wobei Studien mit Ergebnissen, die eine bestimmte Richtung bevorzugen, mit höherer statistischer Signifikanz veröffentlicht werden (Hopewell et al. 2009). Daher kann der Ausschluss von „grauer“, also nicht in referierten Zeitschriften publizierter Literatur zu einer Über- oder Unterschätzung des Effekts führen. Aus diesem Grund werden in der vorliegenden Studie auch Werke aus dieser Sparte gesammelt und auf ihre Tauglichkeit für eine Analyse untersucht.

Schritte 3 und 4 Im Hauptschritt der Datensammlung werden nun die großen akademischen und allgemeinen Datenbanken: Web of Science, Wiley Online Library, Taylor und Francis Online, Science Direct, Emerald Insight, Sage Zeitschriften, Google und Business Source durchsucht. Die allgemeinen Datenbanken Google und Business Source Complete sollten die graue Literatur erfassen. Die acht Datenbanken erforderten drei moderate Anpassungen der beispielhaften Suchsyntax in Tab. 1. Nach den Ergebnissen von Klewitz und Hansen (2014) bilden die ersten Suchergebnisse eine „C-Liste“ von 21.267 Artikeln. Die Titel und Zusammenfassungen der Artikel wurden in der Software Endnote heruntergeladen und manuell überprüft, um die relevanten Artikel weiter in eine „B-Liste“ mit 299 Artikeln einzuordnen. Nach dem Entfernen von 29 Duplikaten ergibt sich nach Überprüfung des vollständigen Textes dieser Artikel auf der Grundlage der Einschluss- und Ausschlusskriterien die „A-Liste“ der 42 wichtigsten Artikel.

Schritt 5 Die endgültige Stichprobe umfasst 42 Studien, die nach folgenden Kriterien codiert wurden: Messung der Energieeffizienz; Methodik; Mustermarkt; Verkaufs- und Mietprämien; und Standardfehler schätzen (Datensatz auf Anfrage vom Autor erhältlich). Alle ausgewählten Studien wurden in Zeitschriften veröffentlicht. 
Tab. 2 Statistische Kennwerte der Primaruntersuchung der Miet- und Kaufpreise

\begin{tabular}{llllllll}
\hline & \multicolumn{7}{l}{ Effektgrösse and Signifikanz } \\
& Koeffizient & Std.fehler & $k$ & Z-Wert & $\tau^{2}$ & Q & $I^{2}$ \\
\hline Verkauf & $0,0761 * * *$ & 0,0179 & 35 & 8,53 & 0,0017 & $1564,75^{* * * *}$ & $97,8 \%$ \\
& {$[0,0586 ; 0,0936]$} & & & & & & {$[97,5 ; 98,1]$} \\
Vermietung & $0,0602 * * *$ & 0,0176 & 31 & 6,84 & 0,0017 & $574,05 * * *$ & $94,8 \%$ \\
& {$[0,0430 ; 0,0775]$} & & & & & & {$[93,5 ; 95,8]$} \\
\hline
\end{tabular}

$95 \%$ Vertrauensintervalle in Klammern $* * * p<0,0001$

Eine Suche in der grauen Literatur ergab keine nicht veröffentlichten Studien, welche die Einschlusskriterien erfüllten. Von den 29 Zeitschriften, in denen die Studien veröffentlicht wurden, erscheinen die einflussreichsten in Energy Policy (4 Studien); Journal of Real Estate Finance and Economics (4 Studien); und Regional Science and Urban Economics (3 Studien).

Die Literatur zu grünen Prämien scheint überwiegend auf die USA ausgerichtet zu sein, insbesondere bei Gewerbeimmobilien (siehe Abb. 2). Dies ist keine Überraschung - die auf dem US-amerikanischen Handelsmarkt verfügbaren Daten weisen bekanntlich die höchste Qualität auf, was die Stichprobengröße und die Anzahl der Variablen angeht (McAllister o.J.). Zudem sind die USA aufgrund ihrer weit verbreiteten Zertifizierungssysteme LEED und Energy Star (Eichholtz et al. 2013) relevanter für eine Studie der Zahlungsbereitschaft als zum Beispiel China, wo lediglich einige wenige Green Building-Initiativen existieren, die zudem staatlich geführt sind (Zheng et al. 2012). In einigen Fällen haben die Autoren von Marktstudien dieses Problem durch die Erstellung von Indizes umgangen, die Immobilien einbeziehen, die in ihren Vermarktungsinformationen Schlagworte wie ,energieeffizient“ und „nachhaltig“ verwenden (z. B. Zheng et al. 2012; Sánchez-Ollero et al. 2014; Aroul und Hansz 2012; Shewmake und Viscusi 2015). Obwohl diese Methode als Heuristik brauchbar sein kann, wenn qualitativ hochwertige Daten fehlen, sind diese Studien dennoch anfällig für Fehler, da die Identifizierung nachhaltiger Objekte lediglich auf Selbstauskünften und Werbematerial der Verkäufer beruht. Dies unterstreicht das bekannte Problem, Immobilienmarktdaten in ausreichender Qualität und Quantität für Studien dieser Art zu erhalten.

\section{Ergebnisse und Diskussion}

Die Primäranalysen der Miet- und Verkaufsprämien, die in Tab. 2 dargestellt sind, ergeben gewichtete mittlere Effekte von 6,0\% bzw. 7,6\%, was auf positive Preiseffekte für die Energieeffizienz und Umweltzertifizierung hindeutet. Die Konfidenzintervalle legen jedoch nahe, dass die wahren mittleren Effekte zwischen 4,3-7,8\% und 5,9-9,4\% liegen, was dem von Morri und Soffietti (2013) erwarteten Bereich entspricht. Diese Schätzungen sind statistisch signifikant. Dies deutet auf eine sichere Ablehnung der Nullhypothese eines Nullmittelwerts (keine grüne Prämie) hin. Da es sich bei den Konfidenzintervallen im Wesentlichen um Normalverteilungen 


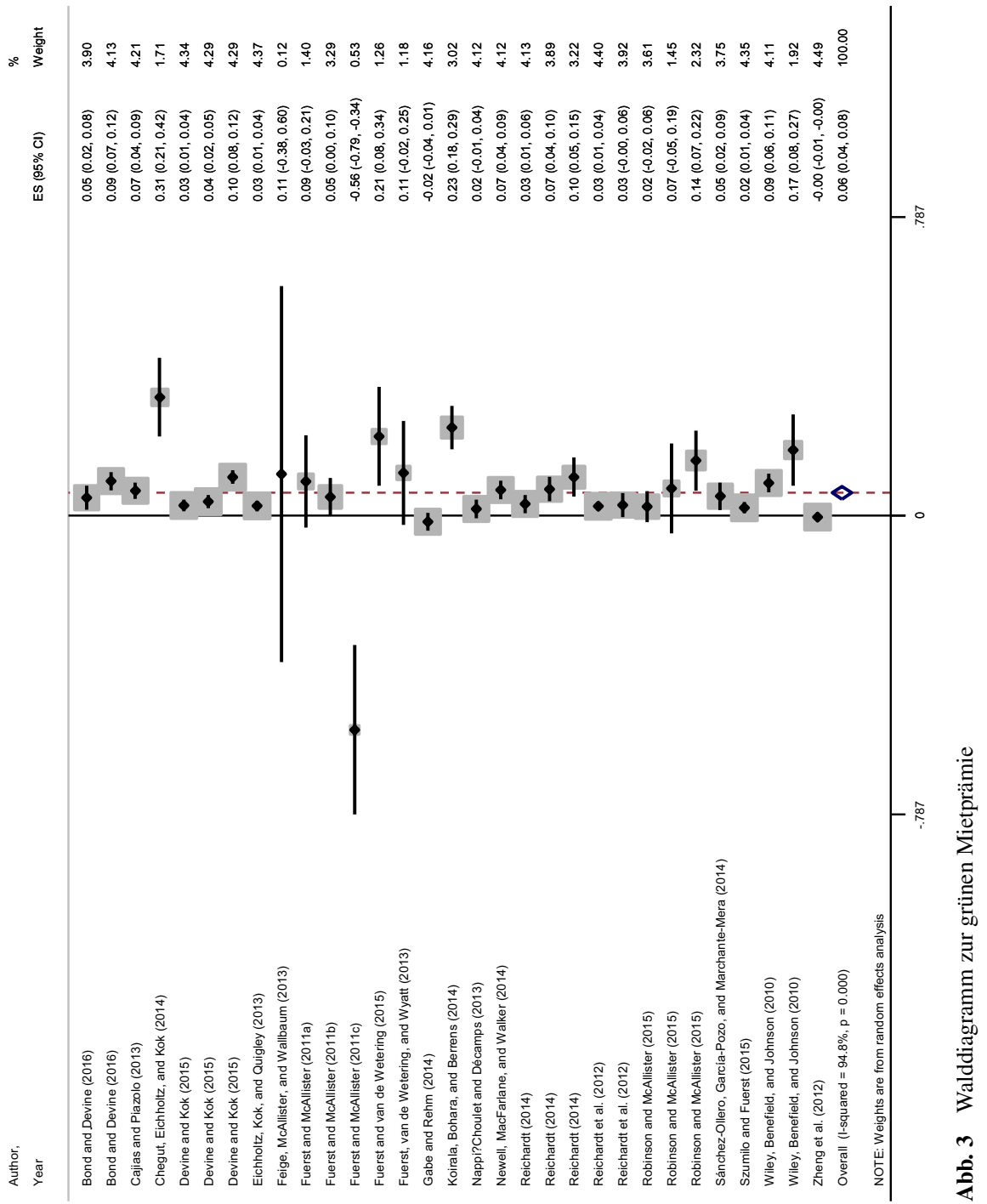




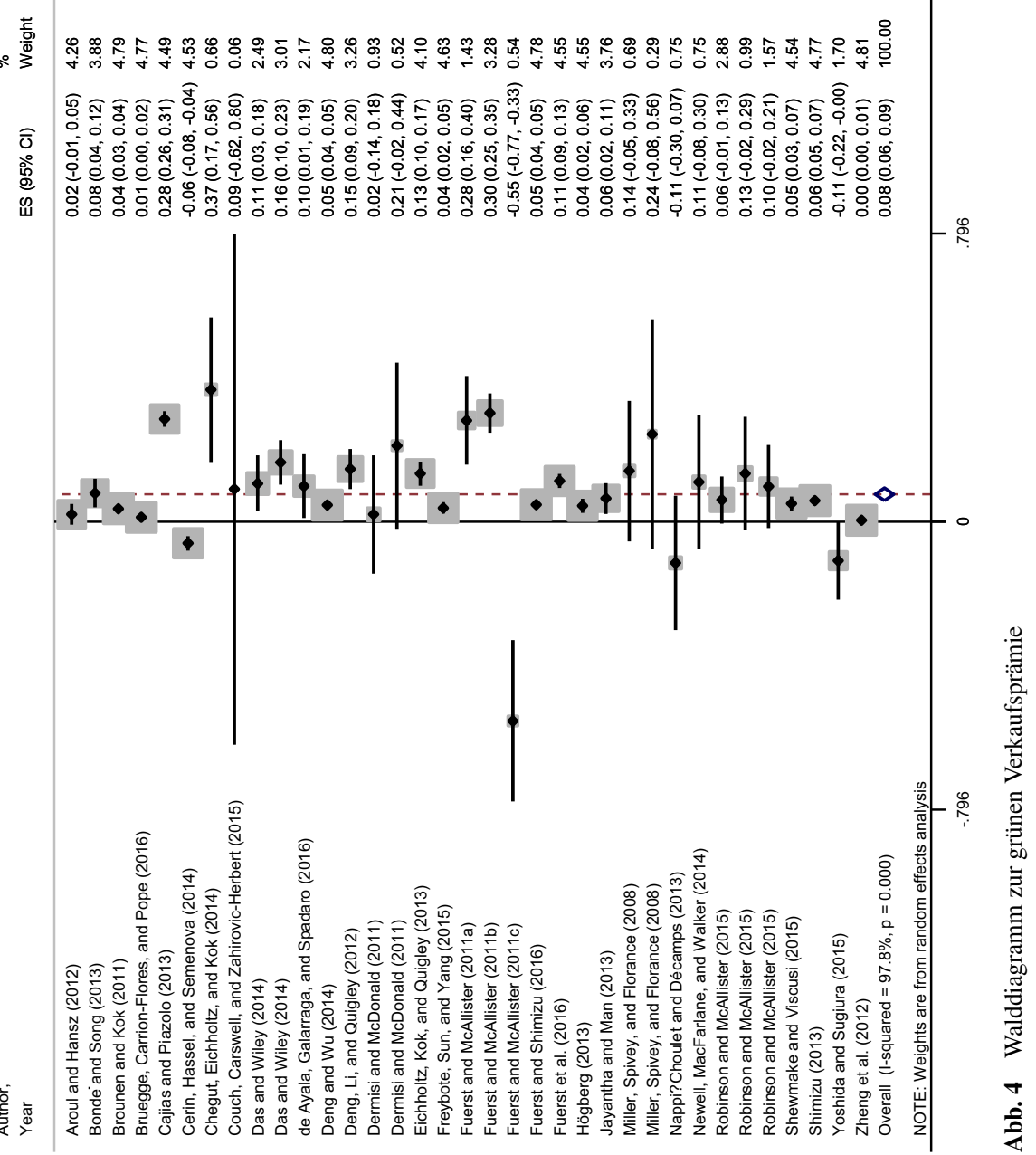




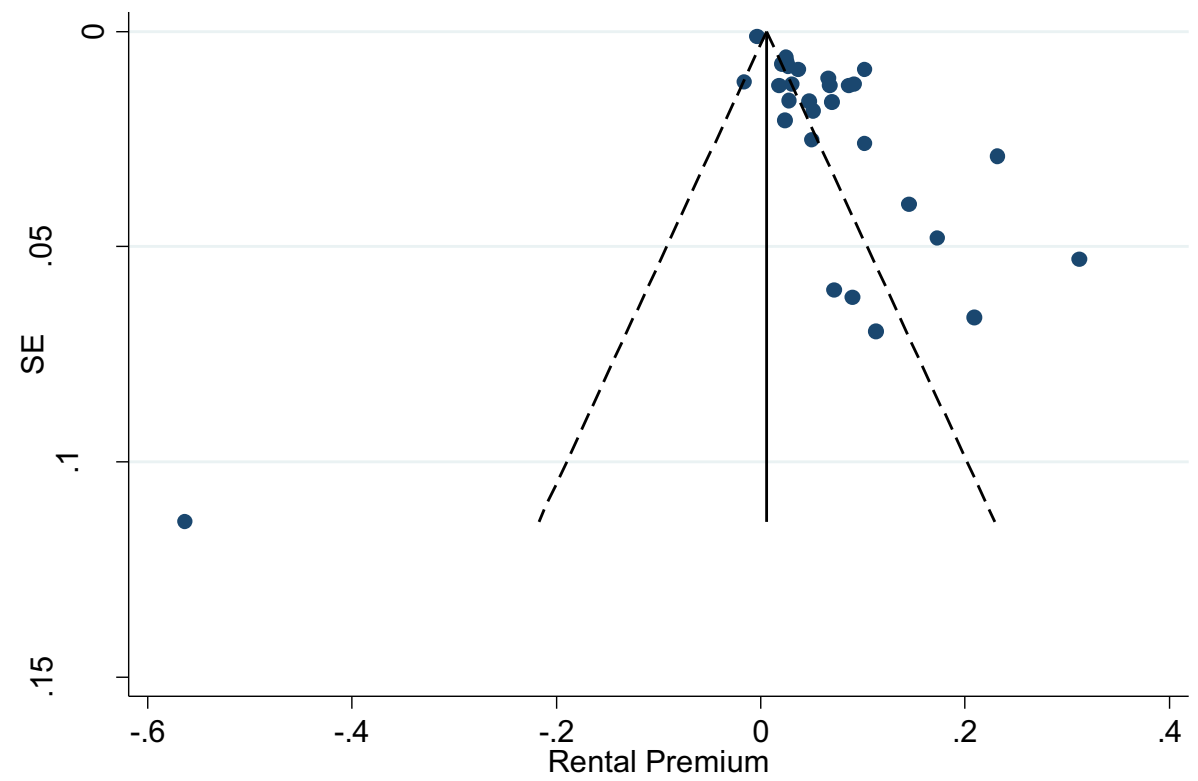

Abb. 5 Trichterplot zur grünen Mietprämie

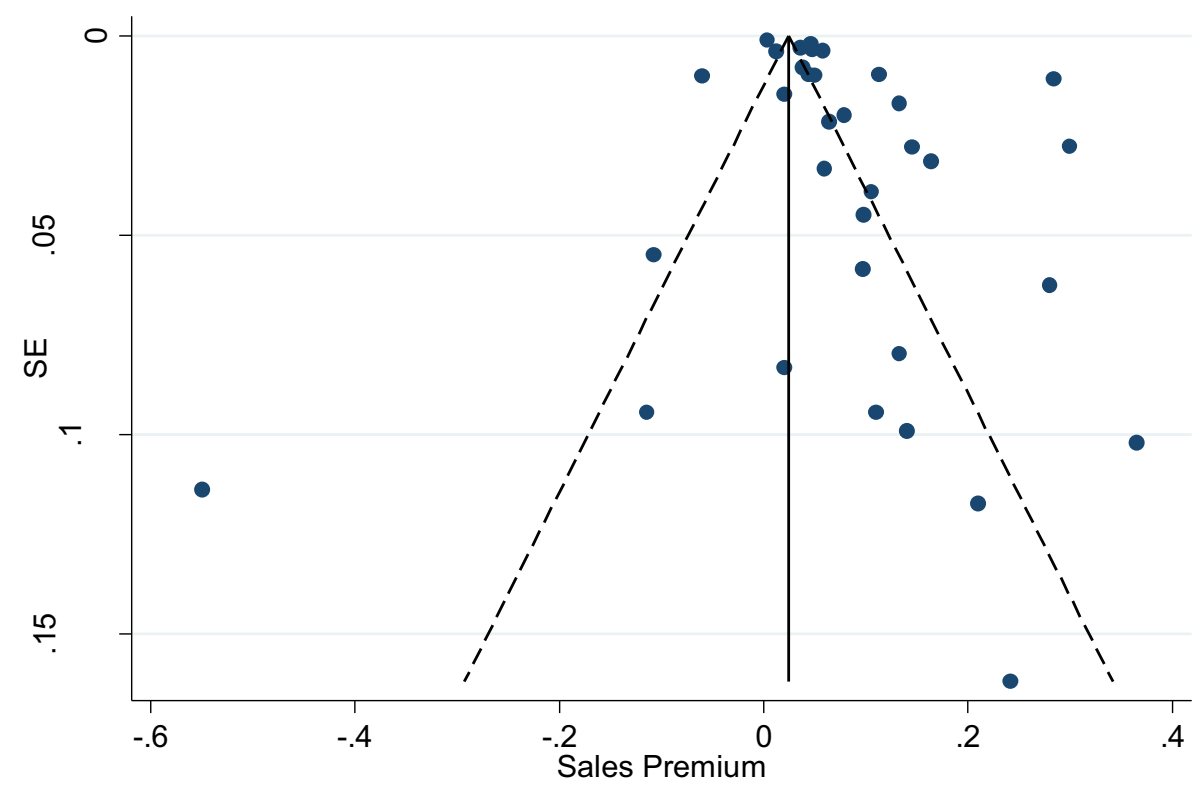

Abb. 6 Trichterplot zur grünen Verkaufsprämie 
Tab. 3 Ergebnis des Egger et al. (1997) Tests

\begin{tabular}{llll}
\hline & $\mathrm{t}$ & $p$ & $95 \% \mathrm{CI}$ \\
\hline Sales & 2,58 & 0,015 & 0,$6779 ; 5,7723$ \\
Rental & 5,53 & 0,000 & 2,$2871 ; 4,9694$ \\
\hline
\end{tabular}

handelt, deutet das Fehlen überlappender Intervalle zwischen den Studien auf eine signifikante Varianz zwischen den Studien $\left(\tau^{2}\right)$ der echten Effekte hin (Borenstein et al. 2011; Cumming und Finch 2005). Somit weist ein signifikanter $\tau^{2}$ auf statistische Heterogenität hin und sollte zu einer Ablehnung der Nullhypothese führen, dass die geprüften Studien einen einheitlichen Effekt schätzen. Als weitere diagnostische Werte werden Cochrans Q sowie der von Higgins (2008) entwickelte I ${ }^{2}$ Index berechnet, nachdem ein Wert über $75 \%$ auf einen hohen Grad an Heterogenität hinweist. Während Heterogenität in einer Metaanalyse unvermeidlich ist, ist die Messung der Größe von Bedeutung (Higgins 2008). Die in Tab. 2 berichteten $\tau 2$, Q und $\mathrm{I}^{2}$-Werte bestätigen, dass bei den Studien zu grünen Prämien ein hoher Grad an Heterogenität der geschätzten Effekte in einzelnen Märkten vorliegt und zwar sowohl bei Verkaufs- als auch bei Vermietungstransaktionen.

Die Ergebnisse der Primäranalysen der Miet- und Verkaufsprämien werden als Walddiagramme (forest plots) dargestellt (Abb. 3 und 4). Die horizontalen Linien zeigen die $95 \%$-Konfidenzintervalle für die beobachteten Effektgrößen. Der Mittelpunkt jeder dieser Linien entspricht der Größe des beobachteten Effekts einer jeweiligen Studie. Die Rauten repräsentieren die geschätzte Gesamteffektgröße und seine Breite repräsentiert ein Vertrauensintervall. der Bereich, außerhalb dessen der wahre Wert unwahrscheinlich ist.

Bei der visuellen Betrachtung zeigt sich, dass die Mehrzahl der in die Analysen einbezogenen Studien einen positiven Preiseffekt mit Ausnahme von drei Mietstudien (Fuerst und McAllister 2011c; Gabe und Rehm 2014; Zheng et al. 2012) und vier Verkaufsstudien (Fuerst und McAllister 2011c; Nappi-Choulet und Décamps 2013; Yoshida und Sugiura 2015; Cerin et al. 2014) feststellt.

Ein möglicher Publikationsbias kann nachfolgend anhand der von Light und Pillemer (1984) vorgeschlagenen Trichterplots ermittelt werden. Die Streudiagramme in den Abb. 5 und 6 zeigen die beobachteten Effekte auf der X-Achse und ihree Standardfehler auf der y-Achse. Die Annahme hinter diesen Plots ist, dass größere Studien niedrigere Standardfehler und eine geringere Streuung bei den auf der x-Achse dargestellten Effekten aufweisen. Sie sind auch gegenüber kleineren Studien stärker symmetrisch verteilt. Mit anderen Worten berichten größere Studien gewöhnlich sowohl positive als auch negative Ergebnisse, wohingegen bei kleineren Studien eher ein Publikationsbias auftreten kann, da bei diesen der Aufwand in der Regel geringer ist und sie daher leichter unpubliziert bleiben können. Die Trichter-Diagramme in Abb. 5 und 6 zeigen sowohl für die Miet- als auch für die Verkaufsprämie eine Asymmetrie gegenüber kleineren Studien auf der x-Achse, so dass möglicherweise Veröffentlichungen in diesem Bereich fehlen (Sterne et al. 2000). Das Fehlen kleinerer Studien zur Schätzung negativer Prämien ist ein Hinweis, aber kein genauer Test (siehe Lau et al. 2006), auf möglichen Publikationsbias oder fehlende bzw zensierte Studien. Der von Egger et al. (1997) entwickelte Test wird verwendet, um die Trichterplotasymmetrie zu testen, was auf eine signifikante Abweichung bei 
Tab. 4 Übersicht der ermittelten grünen Prämien

\begin{tabular}{lllllll}
\hline & Insgesamt & US & Gewerbe & Wohnen & LEED & Energy Star \\
\hline Mietprämie & 6 & 5,9 & 5,4 & 8,2 & 7,3 & 3,6 \\
& {$[4,3-7,5]$} & {$[4,3-7,5]$} & {$[3,7 ; 7,2]$} & {$[2,4-14]$} & {$[4 ; 10,5]$} & {$[1,7 ; 5,5]$} \\
Verkaufsprämie & 7,6 & 10,5 & 11,5 & 5,5 & 8,3 & IS \\
& {$[5,9 ; 9,4]$} & {$[7-14]$} & {$[5,8 ; 17,3]$} & {$[3,6-7,5]$} & {$[2,6 ; 14]$} & \\
\hline
\end{tabular}

IS insignifikant auf $5 \%$ Wahrscheinlichkeitsniveau

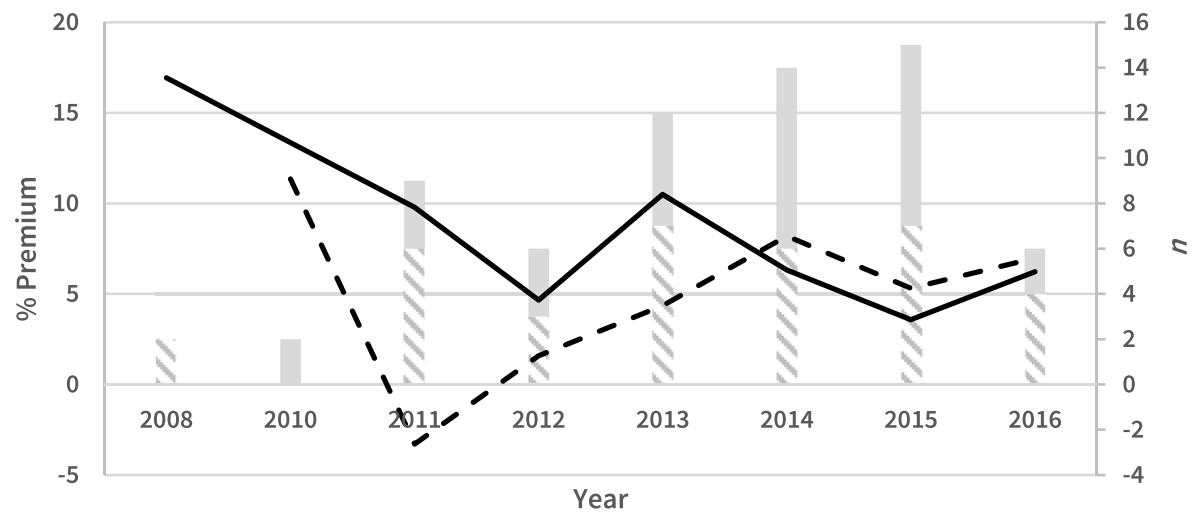

Sales Observations

Rent Observations

Rent Premium - - Sales Premium

Abb. 7 Varianzgewichtete durchschnittliche Prämien nach Publikationsjahr

$5 \%$ hindeutet (siehe Abb. 5 und Tab. 3). Während die Erkennung von Verzerrungen berücksichtigt wird, gibt es keine festgelegte Lösung für das Problem (Sterne et al. 2011).

Während LEED und Energy Star die meisten Beobachtungen bei Mietprämien ausmachen, wird die Verkaufsprämie auch stark von Energieausweisen (EPCs) und anderen Zertifizierungssystemen wie Green Mark und NABERS beeinflusst. Die Untergruppenanalyse der Umsatzprämie nach Energieeffizienzmaßstab schätzt eine signifikante positive Prämie für LEED auf 8,3\% (CI 2,6; 14\%) und andere Zertifizierungssysteme auf 5,3\% (CI 4,1; 6,5\%); Die Konfidenzintervalle für EPCs (CI $-2,5 ; 11,8 \%)$ und Energy Star (CI -0,2; 15,1\%) enthalten jedoch Null, was darauf hinweist, dass ihre Schätzungen keine wesentliche Zurückweisung der Null-Hypothese von Null bedeuten. Während die EPC-Untergruppe eine hohe Heterogenität aufweist, was darauf schließen lässt, dass die Prämie in verschiedenen Ländern in Europa stark variiert, wurde die Energy-Star-Untergruppe ausschließlich in den USA beobachtet und weist geringere Heterogenität auf.

Den beobachteten positiven Preisprämien liegt gemäss den Annahmen dieser Studien eine erhöhte Zahlungsbereitschaft für umweltfreundliche Immobilien zugrunde sowie implizit die Fähigkeit der Käufer, ihre Energieeffizienz und Nachhaltigkeit zumindest halbwegs zuverlässlich einzuschätzen. Vorbehaltlich der Einschränkungen dieser Studie können die erheblichen Prämien, die aus den Metaanalysen der Primär- und der Untergruppe erhalten werden, direkt in Cashflow-Analysen ein- 
fließen, um Investitionsentscheidungen und politische Entscheidungen zu treffen. Die Schätzungen in Tab. 4 zeigen diese Effekte sowie die dazugehörigen positiven Vertrauensintervalle.

Obwohl die oben angegebenen Preisaufschläge statistisch signifikant sind, sollten Investitionsentscheidungen diese nicht als zwingend ansehen. Eine wichtige Einschränkung der zugrundeliegenden Studien, wie von Das und Wiley (2014) angesprochen, besteht darin, dass die geschätzten grünen Prämienkoeffizienten statisch sind. Die meisten Studien berichten nicht, wie sich die Prämie im Laufe der Zeit verändert. In einer der wenigen Studien, die Zeitreihen-Koeffizienten liefern, stellten Reichardt et al. (2012) fest, dass die Energy Star-Verkaufs- und Mietprämien mit der Zeit abgenommen haben.

In Abb. 7 sind die varianzgewichteten Durchschnittswerte der Prämien nach Veröffentlichungsjahr dargestellt. Es ist kein klarer Trend erkennbar. Die Zeitreihe ist zu kurz und die Stichprobe zu klein, um Schlussfolgerungen darüber zu ziehen, ob ein Attrition Effect, also ein Ausfalleffekt durch eine sich systematisch verkleinernde Stichprobe vorliegt. Sollte dieser Effekt jedoch hier tatsächlich vorliegen, bedeutet dies, dass spätere Veröffentlichungen, die längere Zeitreihen verwendeten, die Umweltprämie aufgrund der Verzerrung, die durch die Einbeziehung früherer Studien verursacht wurde, überschätzen. Dieses Phänomen könnte in zukünftigen Studien näher untersucht werden.

\section{Zusammenfassung und Politikempfehlungen}

Die in diesem Beitrag beschriebene Metaanalyse der internationalen Studien zur Rentabilität nachhaltiger Immobilien kommt zu dem Schluss, dass ein relativ starker Konsens besteht, dass als nachhaltig bzw. energieeffizient zertifizierte Gebäude eine zumindest moderate Prämie bei Preisen und Mieten erzielen. Während die Rentabilität im Einzelfall von einer Reihe gebäude- und marktspezifischer Kenngrössen abhängt, liefern diese Studien dennoch einen Anhhaltspunkt dafür, dass nachhaltiges Bauen und Sanieren vom Markt als wirtschaftlich sinnvoll erkannt und entsprechend bepreist wird.

Die Metaanalyse der 42 Studien, welche die Beziehung zwischen Umweltverträglichkeit und Immobilienpreisen untersuchen, beruht auf einer systematischen Überprüfung zweier allgemeiner Datenbanken und sechs wissenschaftlicher Datenbanken. Die Primäranalyse umfasste alle Beobachtungen zur Schätzung einer gewichteten Durchschnittsprämie für Miet- und Verkaufswerte. Die Mietprämie wurde im Durchschnitt auf 6,02\% und die Verkaufsprämie auf 7,61\% geschätzt. Obwohl die Schätzungen beide hochsignifikant waren, zeigten die Analysen eine beträchtliche statistische Heterogenität sowie ein überdurchschnittlich hohes Aufkommen positiver Effekte verglichen mit dem Erwartungswert.

In einem weiteren Schritt wurden Teilgruppen analysiert, um den hohen Grad an Heterogenität zu erklären. Dabei wurden die Effektgrößen sowie die Unterschiede zwischen den Teilgruppen verschiedener Märkte, Energieeffizienzmaßnahmen und Objekttypen betrachtet. Wie erwartet, wurde festgestellt, dass die breite Vielfalt der Studien in allen diesen Bereichen auch signifikant zur Heterogenität der Ergebnisse 
beiträgt. In der immobilienwirtschaftlichen Literatur wird häufig festgestellt, dass Immobilienmärkte stark durch lokale Angebots- und Nachfragestrukturen geprägt, so dass dieses Ergebnis wohl nicht weiter verwundert. Dennoch ist dieses Ergebnis wichtig, da im Gegensatz zu anderen Metaanalysen, etwa im Bereich der Medizin, die auf standardisierte Testverfahren und kontrollierte Umgebungsvariablen zurückgreifen können, ein Teil der Heterogenität der Ergebnisse im Immobilienbereich durch unterschiedliche Marktstrukturen in den untersuchten Städten und Ländern zu erklären ist.

Der potentielle Publikationsbias wurde durch Trichterplotasymmetrie festgestellt und ergab Hinweise auf eine mögliche Verzerrung der Ergebnisse. Würde man diese Verzerrung statistisch korrigieren, ergäbe sich somit wahrscheinlich eine etwas geringere Miet- und Verkaufsprämie nachhaltiger Immobilien.

Unabhängig von der genauen Höhe der zu erzielenden Prämie, stellt sich die Frage, woraus sich die festgestellte erhöhte Zahlungsbereitschaft schöpft. Erhöhte Energieeffizienz führt zu geringeren Betriebskosten und diese Ersparnisse sollten sich entsprechend im Preis der Immobilie niederschlagen. Allerdings beziehen die meisten der hier untersuchten Zertifizierungssysteme eine Reihe weiterer Nachhaltigkeitsfaktoren wie Wasserverbrauch, Luftqualität und Baumaterialien ein. Somit spiegeln die festgestellten Preisprämien neben der Energieeffizien auch diese Faktoren wider. Darüber hinaus werden in der Literatur auch Imagegründe für die erhöhte Zahlungsbereitschaft für nachhaltige Immobilien genannt. Im Bereich der Gewerbeimmobilien ist es für eine wachsende Zahl von Mietern wichtig, firmeninterne Kriterien der ESG (Environmental and Social Governance) sowie CSR (Corporate Social Responsibility) zu erfüllen, die häufig auch mit Auflagen verbunden sind, wie es um die Nachhaltigkeit der von der Firma genutzten Immobilien bestellt ist. Auf dem Wohnungsmarkt sind vergleichbare Effekte zu beobachten. Obschon Haushalte im Gegensatz zu Firmen ihre Umweltziele in der Regel nicht formal beschließen und festhalten, existiert durchaus ein zahlungsstarkes Klientel von Konsumenten, das bereit ist, für „grüne“ Produkte mehr auszugeben und dabei unabhängig von möglichen Betriebskosteneinsparungen entscheiden.

Im deutschen Kontext wird die Debatte um den Mehrwert energieeffizienter und nachhaltiger Immobilien seit Jahren kontrovers geführt. Die Frage nach der Verteilung von Nutzen und Lasten von Nachhaltigkeitsmaßnahmen zwischen Mietern und Vermietern wird vermutlich nirgendwo intensiver geführt als in der Debatte zu Mietsteigerungen nach einer energetischen Sanierung. Hier geht es in erster Linie auch darum, die Sozialverträglichkeit solcher Mietsteigerungen zu gewährleisten. Während hier vor allem die Politik in der Pflicht steht, etwa durch die Gewährung von Subventionen, für einen Ausgleich zu sorgen, stellt sich parallel hierzu das Problem der Split Incentives, also die Tatsache, dass Vermieter Investitionskosten und -risiken tragen, die Mieter aber in der Regel von den niedrigeren Strom- und Heizrechnungen profitieren. Zur Überwindung dieses Problems sind in der Literatur eine Reihe von Vorschlägen gemacht worden, die es zu prüfen gilt. Beispielsweise kann das Split Incentive Problem durch ,grüne Mietverträge“ zumindest abgemildert werden, da es eine Verteilung der Kosten und Nutzen zwischen Mietern und Vermietern festlegt und das Investitionsrisiko mindert. Weitere erfolgversprechende Ansätze sind das Energiespar-Contracting und die Bündelung von Projektrisiken in 
größeren Verbänden, etwa bei Wohnungsgenossenschaften oder auf Quartiersebene (Kholodilin et al. 2016). So ist es auch nicht überraschend, dass eine neuere europäische Vergleichstudie zu Mietrecht und energetischer Sanierung zu dem Ergebnis kommt, dass neben klaren rechtlichen Regeln zur Kostenumlage auch die Strukturen der Mietwohnungsmärkte und hier vor allem starke verbandliche Strukturen und langfristig orientierte Vermieter ausschlaggebend für den energetischen Sanierungszustand des Wohnungsbestands sind (Müller et al. 2016). Da jedoch Instrumente, wie das oben genannte Contracting oder auch grüne Mietverträge noch relativ selten angewandt werden, ist das Hauptergebnis der vorliegenden Metastudie, nämlich dass Nachhaltigkeit in der Regel und unter bestimmten Voraussetzungen, vom Markt erkannt und entlohnt wird, ein wichtiges Signal für Vermieter, dass nachhaltige Investitions- und Modernisierungsentscheidungen sich unter Umständen auch dann lohnen können, wenn der Vermieter die Mehrkosten solcher Maßnahmen zunächst einseitig übernimmt, wobei allerdings die spezifischen Gegebenheiten von Markt und Objekt stets zu berücksichtigen sind.

Danksagung Die Autoren sind folgenden Personen dankbar für Kommentare zu früheren Fassungen: Sara Wilkinson, Sarah Sayce, Norman Miller und Tim Dixon sowie einem anomymen Gutachter dieser Zeitschrift. Die Abbildungen zur Metaanalyse wurden mit freundlicher Genehmigung des Routledge-Verlags in diesem Artikel reproduziert.

Open Access Dieser Artikel wird unter der Creative Commons Namensnennung 4.0 International Lizenz (http://creativecommons.org/licenses/by/4.0/deed.de) veröffentlicht, welche die Nutzung, Vervielfältigung, Bearbeitung, Verbreitung und Wiedergabe in jeglichem Medium und Format erlaubt, sofern Sie den/die ursprünglichen Autor(en) und die Quelle ordnungsgemäß nennen, einen Link zur Creative Commons Lizenz beifügen und angeben, ob Änderungen vorgenommen wurden.

\section{Literatur}

\section{Verwendete Literatur}

Aroul RR, Hansz JA (2012) The value of "green:” evidence from the first mandatory residential green building program. J Real Estate Res 34(1):27-49 (Available at: 〈Go to ISI〉://WOS:000301320900002)

Arup (2014) International sustainability systems comparison. http://publications.arup.com/ /media/ Publications/Files/Publications/I/International_Sustainability_Systems_Report.ashx. Zugegriffen: 12. Januar 2017

Borenstein M, Hedges LV, Higgins JP, Rothstein HR (2011) Introduction to meta-analysis. John Wiley \& Sons, Hoboken, NJ

Brounen D, Kok N (2011) On the economics of energy labels in the housing market. J Environ Econ Manage 62(2):166-179 (http://www.sciencedirect.com/science/article/pii/S0095069611000337)

Brown MJ, Watkins T (2015) The "green premium" for environmentally certified homes: a meta-analysis and exploration. Researchgate Working Paper, 43p.

Cajias M, Fuerst F, Bienert S (2019) Tearing down the information barrier: the price impacts of energy efficiency ratings for buildings in the German rental market. Energy Res Soc Sci 47:177-191

Cerin P, Hassel LG, Semenova N (2014) Energy performance and housing prices. Sust Dev 22(6):404-419. https://doi.org/10.1002/sd.1566

Cumming G, Finch S (2005) Inference by eye. Am Psychol 60(2):170-180

Dalton B, Fuerst F (2018) The 'green value' proposition in real estate. Routledge Handbook of Sustainable Real Estate

Das P, Wiley JA (2014) Determinants of premia for energy-efficient design in the office market. J Prop Res 31(1):64-86. https://doi.org/10.1080/09599916.2013.788543

Deng Y, Li Z, Quigley JM (2012) Economic returns to energy-efficient investments in the housing market: evidence from Singapore. Reg Sci Urban Econ 42(3):506-515 
Dodge Data \& Analytics (2018) World green building trends 2018. Smart market report. Dodge Data \& Analytics, New York

Egger M, Smith GD, Schneider M, Minder C (1997) Bias in meta-analysis detected by a simple, graphical test. BMJ 315(7109):629-634

Eichholtz P, Kok N, Quigley J (2010) Doing well by doing good? Green office buildings. Am Econ Rev 100(5):2492-2509

Eichholtz P, Kok N, Quigley JM (2013) The economics of green building. Rev Econ Stat 95(1):50-63 (Available at: http://search.ebscohost.com/login.aspx?direct=true \&db=bth\&AN=86185656\&site=bsilive)

Fizaine F, Voye P, Baumont C (2018) Les études hédoniques soutiennent-elles une valeur verte élevée dans le bâtiment? Une réponse par la méta-analyse. Rev Econ Polit. Revue D’économie Politique; FAERE Working Paper: Paris, France

Fuerst F, McAllister P (2011a) The impact of energy performance certificates on the rental and capital values of commercial property assets. Energy Policy 39(10):6608-6614 (http://www.sciencedirect. com/science/article/pii/S0301421511006021 [Accessed May 16, 2016])

Fuerst F, Shimizu C (2016) Green luxury goods? The economics of eco-labels in the Japanese housing market. J Jpn Int Econ 39:108-122

Fuerst F, Oikarinen E, Harjunen O (2016) Green signalling effects in the market for energy-efficient residential buildings. Appl Energy 180:560-571

Gabe J, Rehm M (2014) Do tenants pay energy efficiency rent premiums? J Prop Invest Finance 32(4):333-351. https://doi.org/10.1108/JPIF-09-2013-0058

Hamedani AZ, Huber F (2012) A comparative study of DGNB, LEED and BREEAM certificate systems in urban sustainability. In: Pacetti M (Hrsg) The Sustainable City VII: Urban Regeneration and Sustainability, S 121

Higgins JP (2008) Commentary: heterogeneity in meta-analysis should be expected and appropriately quantified. Int J Epidemiol 37(5):1158-1160

Hopewell S, Loudon K, Clarke MJ, Oxman AD, Dickersin K (2009) Publication bias in clinical trials due to statistical significance or direction of trial results. Cochrane Database Syst Rev. https://doi.org/10. 1002/14651858.MR000006.pub3

Kholodilin K, Mense A, Michelsen C (2016) Marktwert der Energieeffizienz: deutliche Unterschiede zwischen Miet- und Eigentumswohnungen. DIW Wochenber 83(28):605-613

Kholodilin KA, Mense A, Michelsen C (2017) The market value of energy efficiency in buildings and the mode of tenure. Urban Stud 54(14):3218-3238

Klewitz J, Hansen EG (2014) Sustainability-oriented innovation of SMEs: a systematic review. J Clean Prod 65:57-75

Lau J, Ioannidis JP, Terrin N, Schmid CH, Olkin I (2006) The case of the misleading funnel plot. BMJ 333(7568):597-600

Light RJ, Pillemer DB (1984) Summing up: the science of reviewing research. Harvard University Press, Cambridge

McAllister P (o. J.) Handle with care? An evaluation of empirical research on the financial returns from investing in real estate assets with superior environmental performance

Morri G, Soffietti F (2013) Greenbuilding sustainability and market premiums in Italy. J Eur Real Est Res 6(3):303-332

Müller R, Schmid C, von Bodelschwingh A, Knorr-Siedow T, Hofmann R, Pinkel T, Wieland S, Keßler O, Jänicke C (2016) Mietrecht und energetische Sanierung im europäischen Vergleich

Nappi-Choulet I, Décamps A (2013) Capitalization of energy efficiency on corporate real estate portfolio value. J Corp Real Estate 15(1):35-52. https://doi.org/10.1108/JCRE-01-2013-0005

Reichardt A, Fuerst F, Rottke N, Zietz J (2012) Sustainable building certification and the rent premium: a panel data approach. J Real Estate Res 34(1):99-126

Shewmake S, Viscusi WK (2015) Producer and consumer responses to green housing labels. Econ Inq 53(1):681-699 (Available at: 〈Go to ISI ://WOS:000345350200039)

Sterne JAC, Egger M, Moher D (2011) Addressing reporting biases. In: Higgins JPT, Green S (Hrsg) Cochrane handbook for systematic reviews of interventions. The Cochrane Collaboration, Hoboken, NJ. handbook.cochrane.org. Zugegriffen: 13. Januar 2017

Sterne JAC, Gavaghan D, Egger M (2000) Publication and related bias in meta-analysis: power of statistical tests and prevalence in the literature. J Clin Epidemiol 53:1119-1129

Sánchez-Ollero JL, García-Pozo A, Marchante-Mera A (2014) How does respect for the environment affect final prices in the hospitality sector? A hedonic pricing approach. Cornell Hosp Q 55(1):31-39 (http:// cqx.sagepub.com/cgi/content/abstract/55/1/31) 
Taltavull de La Paz P, Perez-Sanchez VR, Mora-Garcia RT, Perez-Sanchez JC (2019) Green premium evidence from climatic areas: a case in southern Europe, Alicante (Spain). Sustainability 11(3):686

Yoshida J, Sugiura A (2015) The effects of multiple green factors on condominium prices. J Real Estate Finance Econ 50(3):412-437 (Available at: 〈Go to ISI $\rangle: / / W O S: 000351100800006$ )

Zhang X (2015) Green real estate development in China: State of art and prospect agenda-a review. Renew Sustain Energy Rev 47:1-13

Zheng S, Wu J, Kahn ME, Deng Y (2012) The nascent market for "green" real estate in Beijing. Eur Econ Rev 56(5):974-984

\section{Weiterführende Literatur}

Andelin M, Sarasoja AL, Ventovuori T, Junnila S (2015) Breaking the circle of blame for sustainable buildings-evidence from Nordic countries. J Corp Real Estate 17(1):26-45

Auger CP (1998) Information sources in grey literature (guides to information sources), 4. Aufl. Bowker Saur, London

Borenstein M, Hedges LV, Rothstein HR (2007) meta-analysis: fixed effect vs. random effects. https:// www.meta-analysis.com/downloads/Meta-analysis-fixed-effect-vs-random-effects.pdf. Zugegriffen: 12. Januar 2017

Brundtland GH (1987) Report of the world commission on environment and development: our common future. Oxford University Press, Oxford

Chegut A, Eichholtz P, Kok N (2014) Supply, demand and the value of green buildings. Urban Stud 51(1):22-43 (Available at: 〈Go to ISI〉://WOS:000328600300002)

Department for Communities and Local Government (2014) Improving the energy efficiency of our buildings, London. https://www.gov.uk/government/uploads/system/uploads/attachment_data/file/ 307556/Improving_the_energy_efficiency_of_our_buildings_-_guide_for_the_marketing_sale_ and_let_of_dwellings.pdf. Zugegriffen: 14. Januar 2017

DerSimonian R, Laird N (1986) Meta-analysis in clinical trials. Control Clin Trials 7(3):177-188 (http:// linkinghub.elsevier.com/retrieve/pii/0197245686900462 [Accessed June 17, 2016].)

Freeman ER (1984) Strategic management: a stakeholder perspective. Pitman, Boston

Freybote J, Sun H, Yang X (2015) The impact of LEED neighborhood certification on condo prices. Real Estate Econ 43(3):586-608 (http://search.ebscohost.com/login.aspx?direct=true\&db=bth\& $\mathrm{AN}=108675017$ \& site $=$ bsi-live $)$

Fuerst F, McAllister P, Nanda A, Wyatt P (2015) Does energy efficiency matter to home-buyers? An investigation of EPC ratings and transaction prices in England. Energy Econ 48:145-156

Fuerst F, McAllister P (2011b) Eco-labeling in commercial office markets: Do LEED and Energy Star offices obtain multiple premiums? Ecol Econ 70(6):1220-1230 (http://www.sciencedirect.com/science/ article/pii/S0921800911000565)

Fuerst F, McAllister P (2011c) Green noise or green value? Measuring the effects of environmental certification on office values. Real Estate Econ 39(1):45-69

Hedges LV, Olkin I (1985) Statisical methods for meta-analysis. Academic Press, London

Hedges LV, Vevea JL (1998) Fixed- and random-effects models in meta-analysis. Psychol Methods 3(4):486-504 (http://personal.psc.isr.umich.edu/yuxie-web/files/pubs/Articles/Hedges_Vevea1998. pdf)

Henger R, Voigtländer M (2012) Energetische Modernisierung des Gebäudebestandes: Herausforderungen für private Eigentümer. Studie. Haus \& Grund Deutschland, Köln

Hopewell S, McDonald S, Clarke MJ, Egger M (2007) Grey literature in meta-analyses of randomized trials of health care interventions. In: Cochrane Database of Systematic Reviews (Hrsg) Publication bias in clinical trials due to statistical significance or direction of trial results, Bd. 2, S 2009

Hui EC, Tse C, Yu K (2014) The effect of ISO14001 certification in property management on property price. J Facil Manag 12(2):97-117. https://doi.org/10.1108/JFM-03-2013-0015

Hui EC, Chan EW, Yu K (2015) The effect of LEED certification on Shanghai's prime office rental value. J Facil Manag 13(3):297-310 (http://search.ebscohost.com/login.aspx?direct=true\&db=bth\& $\mathrm{AN}=103192648 \&$ site $=$ bsi-live. $)$

Hui EC, Yu K, Tse C (2016) The impact of environmental management awards and certifications in property management on property price. Facilities 34(5/6):314-338. https://doi.org/10.1108/F-04-20130029 
Jaffe AB, Stavins RN (1994) The energy paradox and the diffusion of conservation technology. Resour Energy Econ 16(2):91-122 (http://scholar.harvard.edu/files/stavins/files/theenergyparadox.ree1994. pdf? $\mathrm{m}=1440012158)$

Kats GH (2003) Green building costs and financial benefits. http://community-wealth.org/sites/clone. community-wealth.org/files/downloads/paper-kats.pdf. Zugegriffen: 13. Januar 2017

Lee WL (2013) A comprehensive review of metrics of building environmental assessment schemes. Energy Build 62:403-413. https://doi.org/10.1016/j.enbuild.2013.03.014

Olubunmi OA, Xia PB, Skitmore M (2016) Green building incentives: a review. Renew Sustain Energy Rev 59:1611-1621

Pivo G (2010) Owner-tenant engagement in sustainable property investing. J Sustain Real Estate 2(1):183-200

Reichardt A (2014) Operating expenses and the rent premium of energy star and LEED certified buildings in the central and eastern U.S. J Real Estate Finance Econ 49(3):413-433

Rosen S (1974) Hedonic prices and implicit markets: product differentiation in pure competition. J Polit Econ 82(1):34-55

Rosenthal R (1979) The "file drawer problem" and tolerance for null results. Psychol Bull 86(3):638-641 (Available at: http://psych.colorado.edu/ willcutt/pdfs/rosenthal_1979.pdf)

Sayce S, Ellison L, Parnell P (2007) Understanding investment drivers for UK sustainable property. Build Res Inf 35(6):629-643

Stanley TD, Doucouliagos H (2012) Meta-regression analysis in economics and business. Routledge, Oxon

Szumilo N, Fuerst F (2015) Who captures the "green value" in the US office market? J Sustain Finance Invest 5(1-2):65-84. https://doi.org/10.1080/20430795.2015.1054336

Van Assen MALM, van Aert RCM, Wicherts JM (2015) Meta-analysis using effect size distribution of only statistically significant studies. Psychol Methods 20(3):293-309

Vimpari J, Junnila S (2014) Value influencing mechanism of green certificates in the discounted cash flow valuation. Int J Strateg Prop Manag 18(3):238-252

Wiley JA, Benefield JD, Johnson KH (2010) Green design and the market for commercial office space. J Real Estate Finance Econ 41(2):228-243 (Available at: 〈Go to ISI〉://WOS:000280074400006) 